\title{
Half-marathoners are younger and slower than marathoners
}

\author{
Beat Knechtle ${ }^{1,2^{*}}$, Pantelis T. Nikolaidis ${ }^{3}$, Matthias A. Zingg ${ }^{2}$, Thomas Rosemann ${ }^{2}$ and Christoph A. Rüst ${ }^{2}$
}

\begin{abstract}
Age and performance trends of elite and recreational marathoners are well investigated, but not for half-marathoners. We analysed age and performance trends in 508,108 age group runners (125,894 female and 328,430 male half-marathoners and 10,205 female and 43,489 male marathoners) competing between 1999 and 2014 in all flat half-marathons and marathons held in Switzerland using single linear regression analyses, mixed-effects regression analyses and analyses of variance. The number of women and men increased across years in both half-marathons and marathons. There were 12.3 times more female half-marathoners than female marathoners and 7.5 times more male half-marathoners than male marathoners. For both half-marathons and marathons, most of the female and male finishers were recorded in age group 40-44 years. In half-marathons, women (10.29 $\pm 3.03 \mathrm{~km} / \mathrm{h})$ were running $0.07 \pm 0.06 \mathrm{~km} / \mathrm{h}$ faster $(p<0.001)$ than men $(10.22 \pm 3.06 \mathrm{~km} / \mathrm{h})$. Also in marathon, women $(14.77 \pm 4.13 \mathrm{~km} / \mathrm{h})$ were running $0.28 \pm 0.16 \mathrm{~km} / \mathrm{h}$ faster $(\mathrm{p}<0.001)$ than men $(14.48 \pm 4.07 \mathrm{~km} / \mathrm{h})$. In marathon, women $(42.18 \pm 10.63$ years $)$ were at the same age than men ( $42.06 \pm 10.45$ years) $(p>0.05)$. Also in half-marathon, women (41.40 \pm 10.63 years) were at the same age than men ( $41.31 \pm 10.30$ years) $(p>0.05)$. However, women and men marathon runners were older than their counterpart half-marathon runners $(p<0.001)$. In summary, $(1)$ more athletes competed in half-marathons than in marathons, (2) women were running faster than men, (3) half-marathoners were running slower than marathoners, and (4) half-marathoners were younger than marathoners.
\end{abstract}

Keywords: Women, Men, Master runner, Age group

\section{Background}

Marathon running is a very popular sport event held all over the world with an increasing number of races and successful finishers over the last years (Ahmadyar et al. 2015; Jokl et al. 2004; Knechtle et al. 2015b; Lehto 2015; Leyk et al. 2007; Lepers and Cattagni 2012). For example, in the USA, there were more than 1200 marathons held in 2014 compared to $\sim 300$ marathons in 2000 (www.runningusa.org/2015-national-runner-survey). The number of successful marathon finishers increased from $\sim 25,000$ in 1976 to the all-time high of $\sim 550,600$ in 2014. However, in the USA, more runners competed in half-marathons than in marathons. The number of halfmarathoners increased from $\sim 303,000$ in 1990 to the alltime high of $\sim 2,046,600$ in 2014. That was, the number of

\footnotetext{
${ }^{*}$ Correspondence: beat.knechtle@hispeed.ch

${ }^{1}$ Gesundheitszentrum St. Gallen, St. Gallen, Switzerland

Full list of author information is available at the end of the article
}

half-marathoners was in 2014 3.7 times higher than the number of marathoners in the USA.

Considering the popularity of half-marathon races, several studies have examined recently many issues (i.e. mostly health-related) in this sport event by comparing it with corresponding trends in full marathon running (De Gonzalo-Calvo et al. 2015; Hart 2013; Jassal et al. 2009; Kim et al. 2012; Reihmane et al. 2013). In a study on the effect of aerobic exercise on systemic inflammation, half-marathoners showed lower levels of inflammatory parameters after the race compared to marathoners (De Gonzalo-Calvo et al. 2015). In addition, it has been shown that the increase in interleukin-6, tumour necrosis factor-alpha and matrix metalloproteinase-9 after the race was smaller in half-marathoners than in marathoners (Reihmane et al. 2013). In a study on the effect of aerobic exercise on cardiac injury markers, half-marathoners demonstrated lower elevations in creatinine kinase, myoglobin and cardiac troponin $\mathrm{T}$ compared to

\section{Springer}

(c) 2016 Knechtle et al. This article is distributed under the terms of the Creative Commons Attribution 4.0 International License (http://creativecommons.org/licenses/by/4.0/), which permits unrestricted use, distribution, and reproduction in any medium, provided you give appropriate credit to the original author(s) and the source, provide a link to the Creative Commons license, and indicate if changes were made. 
marathoners (Jassal et al. 2009). Moreover, the overall incidence of cardiac arrests in the USA from 2000 to 2010 was lower in half-marathoners than in marathoners (Hart 2013; Kim et al. 2012). These studies focusing on healthrelated aspects have highlighted certain differences in the response of various physiological mechanisms to aerobic exercise between half-marathon and marathon running.

The abovementioned studies on differences between half-marathoners and marathoners have enhanced our understanding of the responses of certain physiological mechanisms to aerobic exercise of various durations. However, only a few data are available about major aspects (e.g. age, sex and race speed) related to performance differences between these two popular running events (Leyk et al. 2007; Zillmann et al. 2013). These studies investigated only a limited time frame or a limited sample of athletes. Leyk et al. (2007) analysed race times and ages of half-marathoners and marathoners for 3 years (2002-2005) and Zillmann et al. (2013) performed a field study on male half-marathoners and marathoners. The knowledge of half-marathon runners' basic characteristics such as age, sex, participation and performance trends might help coaches, fitness trainers and sports scientists to improve their understanding of half-marathon's demands compared to the corresponding profile of a full marathon. Therefore, the aim of this study was to compare age, sex, participation and performance between female and male half-marathoners and marathoners in a sample of more than 500,000 runners competing in half-marathons and marathons held in one country during a period of 15 years.

\section{Materials and methods}

\section{Ethics approval}

The study was approved by the Institutional Review Board of St. Gallen, Switzerland, with waiver of the requirement for informed consent given that the study involved the analysis of publicly available data.

\section{Data sampling and data analysis}

All marathons and half-marathons held in Switzerland were identified by using data from 'Laufkalender Schweiz' (www.laufkalender.ch). In Switzerland, all running races started in 1999 to record race times with an electronic chip system and all race results became available in this year on the websites of the specific races. Of all recorded races, only those half-marathons and marathons were considered which were held on a road, not on a trail. Only flat marathons were considered and no mountain marathons were included. For all considered races, start and finish had to be on the same altitude. Athletes with missing age were excluded from data analysis. In order to avoid a selection bias due to a limitation to top runners (e.g. annual fastest, annual ten fastest), we considered all finishers. Race times recorded in the ranking lists were converted to running speed $(\mathrm{km} / \mathrm{h})$ using race distance $(\mathrm{km})$ and race time (h:min).

\section{Statistical analysis}

Each set of data was tested for normal distribution (D'Agostino and Pearson omnibus normality test) and for homogeneity of variances (Levene's test) before statistical analyses. Differences in the participation of long-distance runners by sex to half-marathon and marathon running were examined using Chi square $\left(x^{2}\right)$ test. Trends in participation across calendar years were analyzed using regression with linear growth equation models. A mixedeffects regression model with finisher as random variable to consider finishers who completed several races was used to analyze changes in performance of finishers across years. We included sex, centered age, squared centered age and calendar year as fixed variables. Sex difference was calculated as sex difference $=$ (running speed in women - running speed in men)/running speed in men $\times 100$, where running speed in men was defined as $100 \%$. Multiple groups were compared using one-way analysis of variance (ANOVA) with subsequent Tukey's post hoc multiple comparison test, with a single pooled variance. Statistical analyses were performed using IBM SPSS Statistics (Version 22, IBM SPSS, Chicago, IL, USA) and GraphPad Prism (Version 6.01, GraphPad Software, La Jolla, CA, USA). Significance was accepted at $\mathrm{p}<0.05$ (two-sided for $t$-tests). Data in the text are given as mean \pm standard deviation (SD). Data in the figures are given as mean $\pm 95 \%$ confidence interval (CI) for boxwhisker-plots and mean \pm SD for trends across time.

\section{Results}

Data from a total of 508,108 (i.e. 125,894 female and 328,430 male half-marathoners and 10,205 female and 43,489 male marathoners) athletes could be considered. There were 12.3 times more female half-marathoners than female marathoners and 7.5 times more male half-marathoners than male marathoners. There was a statistically significant association between the sex of long-distance runners and the format of the race $\left[\mathrm{X}^{2}(1)>40.35 \times 10^{6}, \mathrm{p}<0.001\right]$. That was, compared to men, women participated more in half-marathon than in marathon running.

\section{Participation}

In half-marathons, the number of women $\left(\mathrm{r}^{2}=0.98\right.$, $\mathrm{p}<0.0001)$ and men $\left(\mathrm{r}^{2}=0.98, \mathrm{p}<0.0001\right)$ increased significantly. Similarly, the number of women $\left(r^{2}=0.46\right.$, $\mathrm{p}=0.0041)$ and men increased significantly $\left(\mathrm{r}^{2}=0.51\right.$, $\mathrm{p}=0.0019$ ) in marathons (Table 1 ). The men-to-women 
Table 1 The number of female and male finishers in halfmarathon and marathon

\begin{tabular}{cccccc}
\hline Year & \multicolumn{2}{c}{ Half-marathon } & & \multicolumn{2}{c}{ Marathon } \\
\cline { 2 - 3 } & Women & Men & & Women & Men \\
\hline 1999 & 1674 & 6093 & & 156 & 512 \\
2000 & 2704 & 9793 & & 174 & 807 \\
2001 & 3283 & 10,104 & & 282 & 923 \\
2002 & 4321 & 12,882 & & 255 & 905 \\
2003 & 5057 & 15,178 & & 542 & 2485 \\
2004 & 6537 & 17,275 & & 658 & 2866 \\
2005 & 6704 & 17,249 & & 844 & 3648 \\
2006 & 7233 & 18,583 & & 781 & 3139 \\
2007 & 6686 & 20,879 & & 1073 & 4346 \\
2008 & 9180 & 23,254 & & 992 & 3918 \\
2009 & 10,625 & 25,450 & & 879 & 3908 \\
2010 & 10,332 & 27,675 & & 680 & 3453 \\
2011 & 11,782 & 30,035 & & 785 & 3453 \\
2012 & 11,615 & 27,700 & & 693 & 3126 \\
2013 & 13,825 & 32,555 & & 692 & 3199 \\
2014 & 14,336 & 33,725 & & 719 & 2801 \\
Total & 125,894 & 328,430 & 10,205 & 43,489 \\
\hline
\end{tabular}

ratio decreased significantly in half-marathons $\left(\mathrm{r}^{2}=0.71\right.$, $\mathrm{p}<0.0001)$ but remained unchanged in marathons $\left(r^{2}=0.21, p=0.075\right)$ (Table 2). For both half-marathons and marathons, most female and male finishers were recorded in age group 40-44 years (Table 3).

Table 2 The men-to-women ratio for half-marathon and marathon

\begin{tabular}{lll}
\hline Year & Half-marathon & Marathon \\
\hline 1999 & 3.63 & 3.28 \\
2000 & 3.62 & 4.63 \\
2001 & 3.07 & 3.27 \\
2002 & 2.98 & 3.54 \\
2003 & 3.00 & 4.58 \\
2004 & 2.64 & 4.35 \\
2005 & 2.57 & 4.32 \\
2006 & 2.56 & 4.01 \\
2007 & 3.12 & 4.05 \\
2008 & 2.53 & 3.94 \\
2009 & 2.39 & 4.44 \\
2010 & 2.67 & 5.07 \\
2011 & 2.54 & 4.39 \\
2012 & 2.38 & 4.51 \\
2013 & 2.35 & 4.62 \\
2014 & 2.35 & 3.89 \\
\hline
\end{tabular}

Table 3 Distribution of the athletes regarding the age groups

\begin{tabular}{|c|c|c|c|c|}
\hline \multirow[t]{2}{*}{ Age group } & \multicolumn{2}{|c|}{ Half-marathon } & \multicolumn{2}{|c|}{ Marathon } \\
\hline & Women & Men & Women & Men \\
\hline $18-24$ & 5709 & 15,231 & 405 & 1632 \\
\hline $25-29$ & 11,111 & 29,003 & 859 & 3507 \\
\hline $30-34$ & 16,343 & 43,109 & 1255 & 5516 \\
\hline $35-39$ & 20,796 & 55,542 & 1598 & 7100 \\
\hline $40-44$ & 24,286 & 62,976 & 1967 & 8461 \\
\hline $45-49$ & 20,839 & 53,616 & 1653 & 7232 \\
\hline $50-54$ & 13,619 & 34,992 & 1204 & 4918 \\
\hline $55-59$ & 7458 & 19,072 & 664 & 2685 \\
\hline $60-64$ & 3652 & 9419 & 344 & 1434 \\
\hline $65-69$ & 1460 & 3865 & 173 & 639 \\
\hline $70-74$ & 482 & 1216 & 67 & 271 \\
\hline $75-79$ & 114 & 324 & 14 & 74 \\
\hline $80-84$ & 19 & 52 & 2 & 13 \\
\hline $85-89$ & 4 & 11 & & 6 \\
\hline $90-94$ & 2 & 2 & & 1 \\
\hline
\end{tabular}

\section{Performance}

Figure 1 shows the box-whisker-plots for running speed for female and male half-marathoners and marathoners. In half-marathons, women were running at $10.29 \pm 3.03 \mathrm{~km} / \mathrm{h}$ and men at $10.22 \pm 3.06 \mathrm{~km} / \mathrm{h}$. Women were running $0.07 \pm 0.06 \mathrm{~km} / \mathrm{h}$ faster than men $(\mathrm{p}<0.001)$. Female marathoners were running at $14.77 \pm 4.13 \mathrm{~km} / \mathrm{h}$ and male marathoners at $14.48 \pm 4.07 \mathrm{~km} / \mathrm{h}$. Women were running $0.28 \pm 0.16$ faster than men $(\mathrm{p}<0.001)$. When marathoners and half-marathoners were compared, female marathoners were running $4.47 \pm 1.12 \mathrm{~km} / \mathrm{h}$ faster than female half-marathoners $(\mathrm{p}<0.001)$ and male marathoners were running $4.26 \pm 0.99 \mathrm{~km} / \mathrm{h}$ faster than male half-marathoners $(\mathrm{p}<0.001)$.

Running speed decreased significantly across years in female half-marathoners $\left(\mathrm{r}^{2}=0.55, \mathrm{p}=0.0010\right)$, but remained unchanged in male half-marathoners $\left(r^{2}=0.05, p=0.38\right)$ (Fig. 2a). In female $\left(r^{2}=0.00\right.$, $\mathrm{p}=0.80)$ and male $\left(\mathrm{r}^{2}=0.24, \mathrm{p}=0.051\right)$ marathoners, running speed remained unchanged (Fig. 2b).

Regarding running speed for female (Table 4) and male (Table 5) age group half-marathoners, running speed decreased significantly in age groups 25-29 to 55-59 years (Table 6). Women were faster than men in age groups $25-29$ to $35-39,45-49$ and $50-54$ years. In marathon races (Tables 7,8 ), running speed increased significantly in age group $80-84$ years (Table 9). Women were faster than men in age groups $40-44,50-54$ and 55-59 years. 


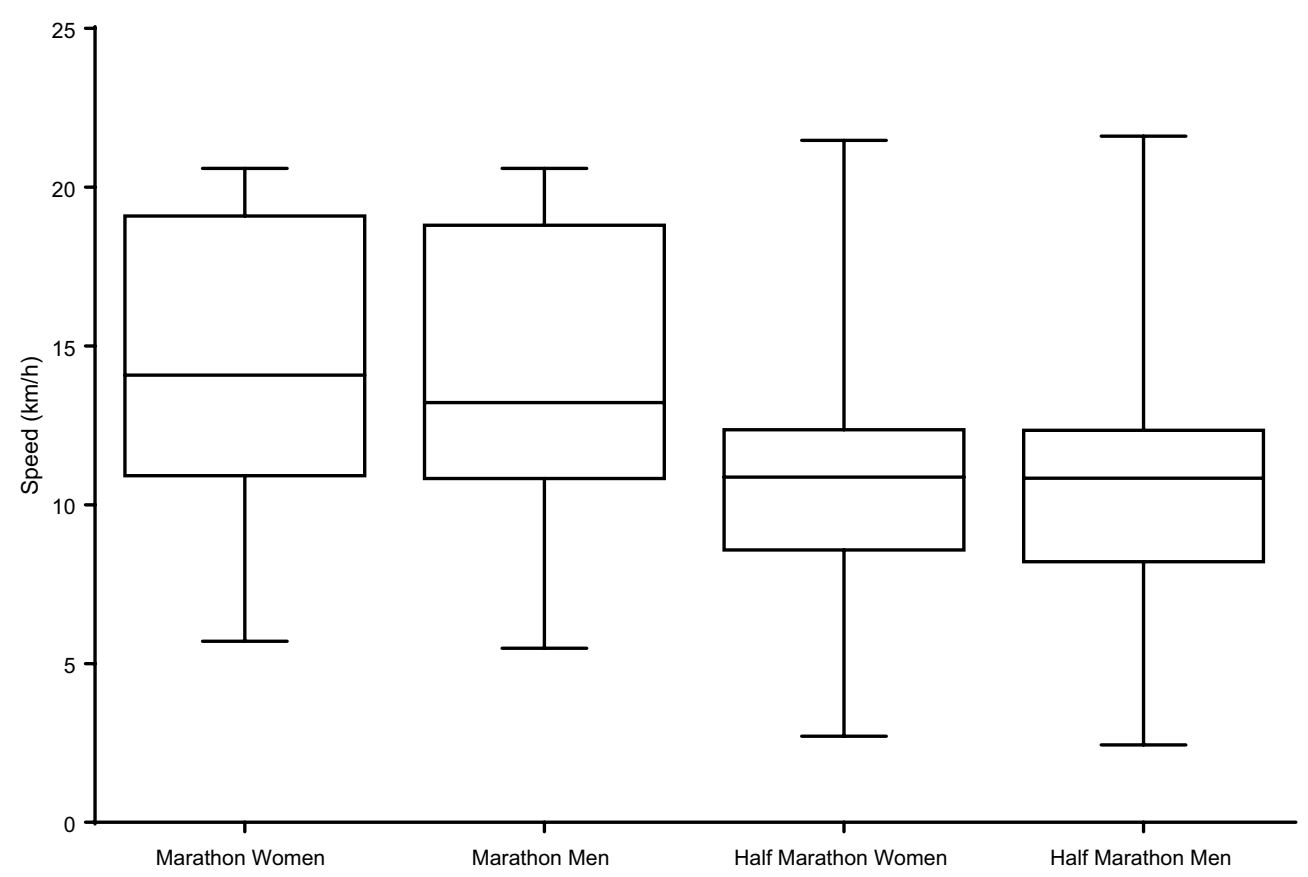

Fig. 1 Box-whisker-plot for running speeds in female and male half-marathoners and marathoners. Data are presented as mean $\pm 95 \%$ confidence interval
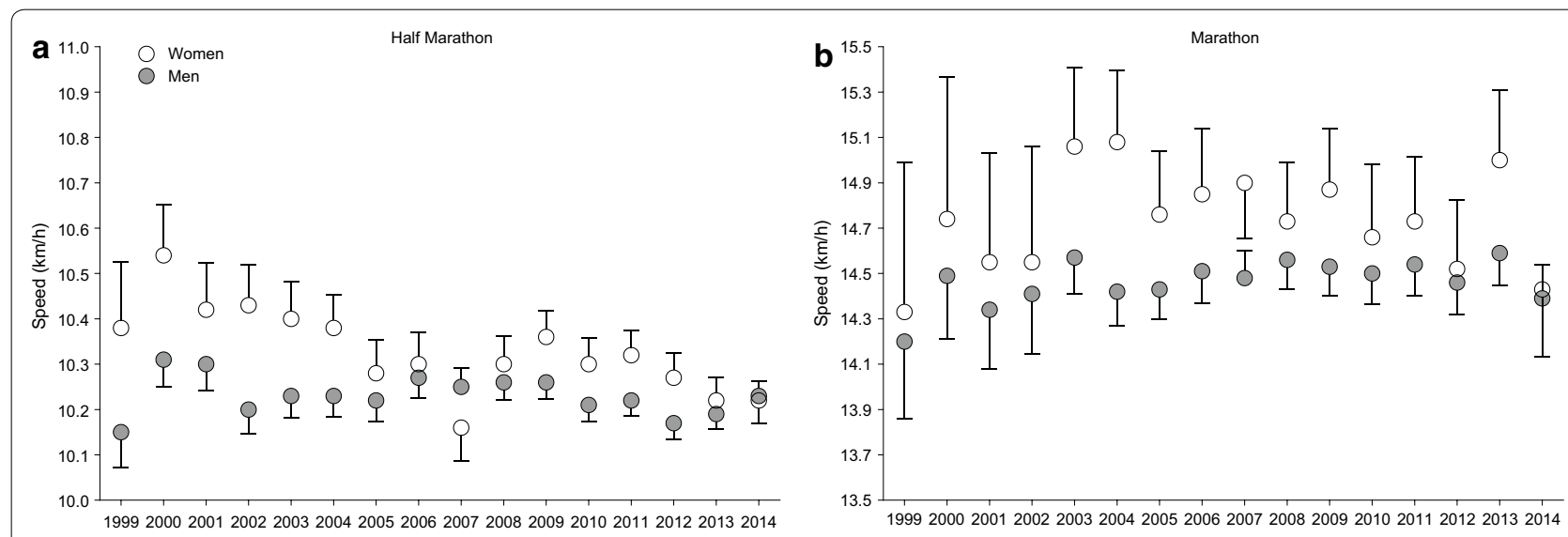

Fig. 2 Running speed across years in half-marathoners and marathoners. Data are presented as mean \pm SD

Sex difference in running speed decreased significantly in half-marathon running in age groups 35-39 and $50-54$ years (Table 10) and in marathon running in age group 45-49 years (Table 11). For all other age groups, sex difference remained unchanged across years.

\section{Age}

Figure 3 shows the box-whisker-plots for age for female and male half-marathoners and marathoners. In marathoners, women $(42.18 \pm 10.63$ years $)$ were at the same age than men $(42.06 \pm 10.45$ years $)(p>0.05)$. Similarly, in half-marathoners, women $(41.40 \pm 10.63$ years $)$ were at the same age than men $(41.31 \pm 10.30$ years $)$ ( $\mathrm{p}>0.05)$. However, women in marathon running were $0.78 \pm 0.33$ years older than women in half-marathon running $(\mathrm{p}<0.001)$ and men in marathon running were $0.75 \pm 0.14$ years older than women in half-marathon running $(\mathrm{p}<0.001)$.

Figure 4 shows trend in age of half-marathoners and marathoners across years. In female $\left(r^{2}=0.00, p=0.93\right)$ and male $\left(\mathrm{r}^{2}=0.12, \mathrm{p}=0.18\right)$ marathoners, age remained unchanged. Similarly, in female $\left(\mathrm{r}^{2}=0.12, \mathrm{p}=0.19\right)$ 


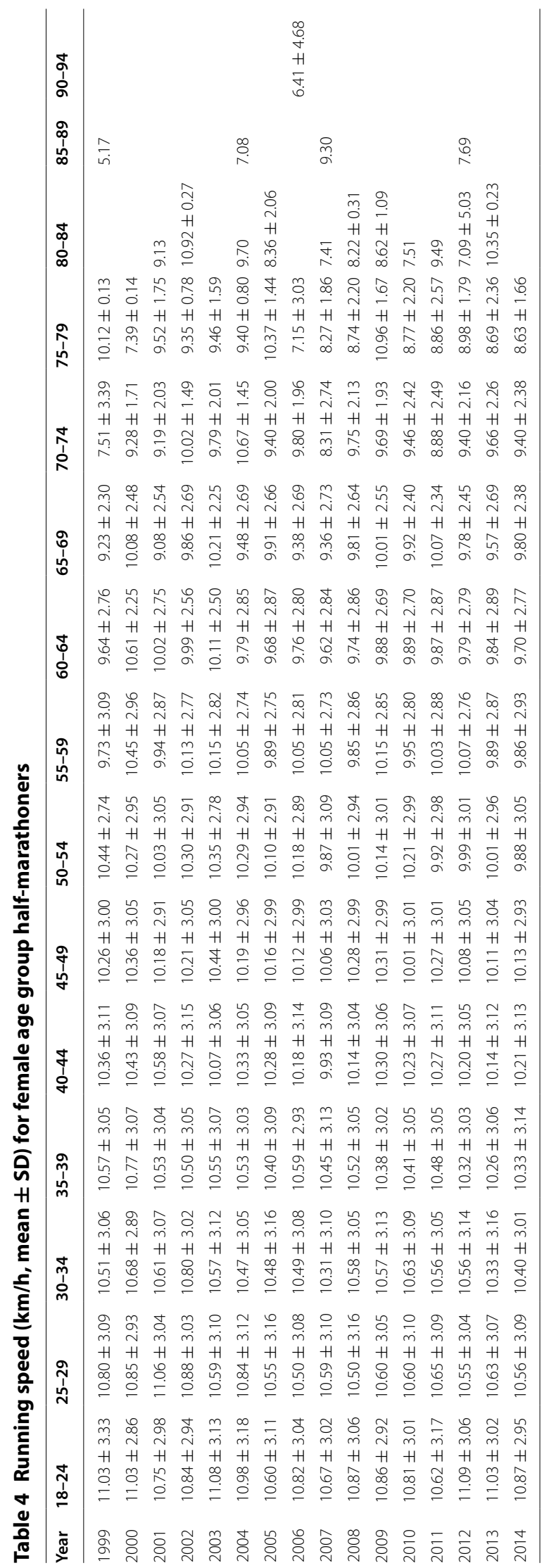




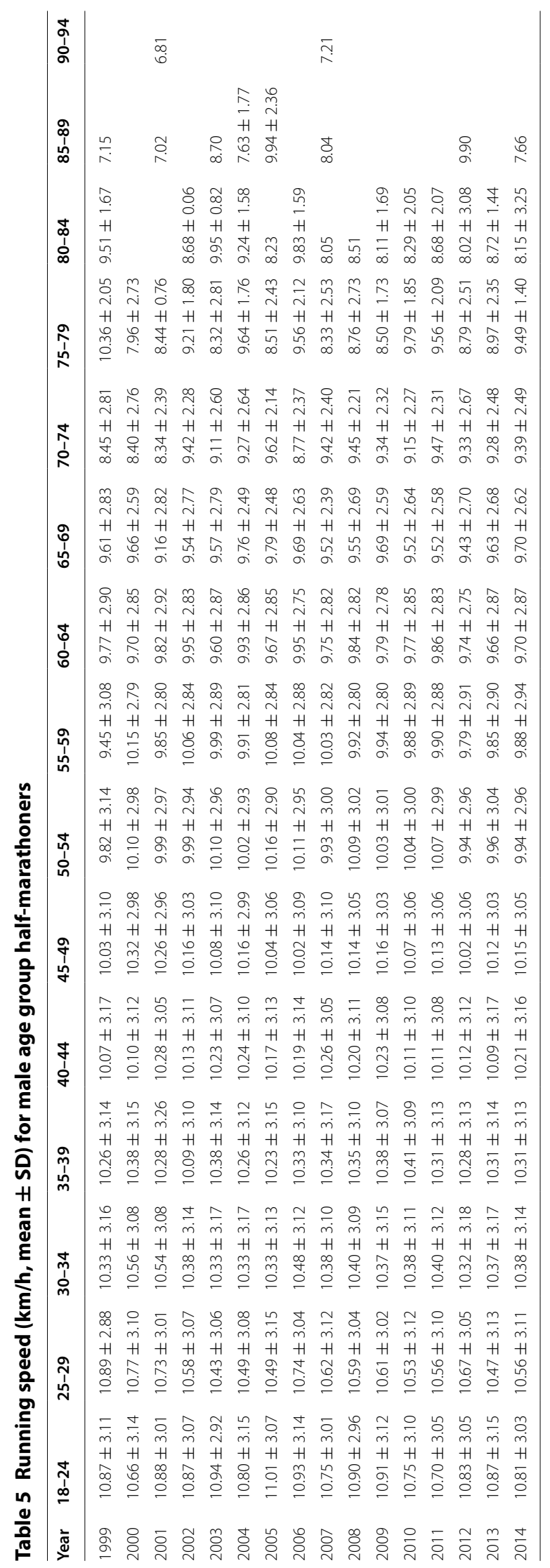


Table 6 Results of the mixed-effects regression analyses for running speed in half-marathon

\begin{tabular}{|c|c|c|c|c|c|}
\hline Parameter & Estimate & SE & df & $t$ & $p$ \\
\hline \multicolumn{6}{|l|}{ 18-24 years } \\
\hline Constant term & 6.296428 & 9.049231 & $16,363.659$ & 0.696 & 0.487 \\
\hline$[\mathrm{Sex}=$ female $]$ & 0.049958 & 0.052227 & $16,454.169$ & 0.957 & 0.339 \\
\hline Calendar year & -0.001075 & 0.004398 & $16,470.927$ & -0.244 & 0.807 \\
\hline Cage & -0.672955 & 0.196899 & $12,995.658$ & -3.418 & 0.001 \\
\hline Cage $^{2}$ & -0.016791 & 0.004985 & $13,014.387$ & -3.369 & 0.001 \\
\hline \multicolumn{6}{|l|}{ 25-29 years } \\
\hline Constant term & 26.794555 & 6.117458 & $28,544.800$ & 4.380 & $<0.0001$ \\
\hline$[$ Sex $=$ female $]$ & 0.122531 & 0.039147 & $29,404.862$ & 3.130 & 0.002 \\
\hline Calendar year & -0.007846 & 0.002974 & $28,838.319$ & -2.638 & 0.008 \\
\hline Cage & 0.092807 & 0.187007 & $22,771.577$ & 0.496 & 0.620 \\
\hline Cage $^{2}$ & 0.004071 & 0.006706 & $22,735.366$ & 0.607 & 0.544 \\
\hline \multicolumn{6}{|l|}{ 30-34 years } \\
\hline Constant term & 12.205098 & 4.717605 & $40,495.293$ & 2.587 & 0.010 \\
\hline$[$ Sex $=$ female $]$ & 0.130067 & 0.033106 & $41,901.765$ & 3.929 & $<0.0001$ \\
\hline Calendar year & -0.001042 & 0.002342 & $40,566.839$ & -0.445 & 0.656 \\
\hline Cage & -0.081075 & 0.092362 & $32,386.180$ & -0.878 & 0.380 \\
\hline Cage $^{2}$ & -0.005457 & 0.005138 & $32,359.413$ & -1.062 & 0.288 \\
\hline \multicolumn{6}{|l|}{$35-39$ years } \\
\hline Constant term & 10.270803 & .028546 & $65,078.789$ & 359.801 & $<0.0001$ \\
\hline$[$ Sex $=$ female $]$ & .243288 & .057024 & $66,997.713$ & 4.266 & $<0.0001$ \\
\hline Calendar year & -.015718 & .006081 & $41,539.469$ & -2.585 & 0.010 \\
\hline Cage & .038838 & .012498 & $48,772.947$ & 3.108 & 0.002 \\
\hline Cage $^{2}$ & -.015916 & .004749 & $58,942.732$ & -3.351 & 0.001 \\
\hline \multicolumn{6}{|l|}{ 40-44 years } \\
\hline Constant term & 9.125955 & 3.973687 & $52,292.011$ & 2.297 & 0.022 \\
\hline$[$ Sex $=$ female $]$ & 1.718455 & 8.450069 & $63,836.054$ & 0.203 & 0.839 \\
\hline Calendar year & 0.000530 & 0.001978 & $52,290.339$ & 0.268 & 0.789 \\
\hline Cage & -0.011926 & 0.005323 & $45,919.163$ & -2.240 & 0.025 \\
\hline Cage $^{2}$ & -0.000813 & 0.004207 & $63,833.556$ & -0.193 & 0.847 \\
\hline \multicolumn{6}{|l|}{$45-49$ years } \\
\hline Constant term & 14.392008 & 3.666324 & $45,133.538$ & 3.925 & $<0.0001$ \\
\hline$[$ Sex $=$ female $]$ & 0.111541 & 0.028930 & $50,970.153$ & 3.856 & $<0.0001$ \\
\hline Calendar year & -0.002073 & 0.001825 & $45,147.299$ & -1.136 & 0.256 \\
\hline Cage & -0.011505 & 0.047202 & $36,973.118$ & -0.244 & 0.807 \\
\hline Cage $^{2}$ & -0.000515 & 0.003940 & $36,937.873$ & -0.131 & 0.896 \\
\hline \multicolumn{6}{|l|}{ 50-54 years } \\
\hline Constant term & 21.134855 & 4.648196 & $28,090.963$ & 4.547 & $<0.0001$ \\
\hline$[$ Sex $=$ female $]$ & 0.081346 & 0.034127 & $35,679.933$ & 2.384 & 0.017 \\
\hline Calendar year & -0.005912 & 0.002295 & $28,200.348$ & -2.576 & 0.010 \\
\hline Cage & 0.142107 & 0.110024 & $22,499.549$ & 1.292 & 0.197 \\
\hline Cage $^{2}$ & -0.006415 & 0.005025 & $22,452.308$ & -1.277 & 0.202 \\
\hline \multicolumn{6}{|l|}{$55-59$ years } \\
\hline Constant term & 14.483520 & 6.509904 & $14,441.900$ & 2.225 & 0.026 \\
\hline$[$ Sex $=$ female $]$ & 0.064112 & 0.042767 & $21,011.361$ & 1.499 & 0.134 \\
\hline Calendar year & -0.003629 & 0.003124 & $14,741.530$ & -1.162 & 0.245 \\
\hline Cage & .381976 & 0.217497 & $10,973.387$ & 1.756 & 0.079 \\
\hline Cage $^{2}$ & -0.013001 & 0.006831 & $10,958.017$ & -1.903 & 0.057 \\
\hline
\end{tabular}


Table 6 continued

\begin{tabular}{|c|c|c|c|c|c|}
\hline Parameter & Estimate & SE & df & $\mathrm{t}$ & $p$ \\
\hline \multicolumn{6}{|l|}{ 60-64 years } \\
\hline Constant term & 10.330497 & 10.184571 & 7038.354 & 1.014 & 0.310 \\
\hline$[$ Sex $=$ female $]$ & 0.066517 & 0.059201 & $10,801.639$ & 1.124 & 0.261 \\
\hline Calendar year & -0.001449 & 0.004592 & 7487.328 & -0.315 & 0.752 \\
\hline Cage & 0.240891 & 0.427255 & 5330.091 & 0.564 & 0.573 \\
\hline Cage $^{2}$ & -0.006042 & 0.010224 & 5320.416 & -0.591 & 0.555 \\
\hline \multicolumn{6}{|l|}{$65-69$ years } \\
\hline Constant term & -21.260625 & 17.377053 & 2734.553 & -1.223 & 0.221 \\
\hline$[$ Sex $=$ female $]$ & 0.171801 & 0.083333 & 4650.236 & 2.062 & 0.390 \\
\hline Calendar year & 0.010878 & 0.006919 & 3161.386 & 1.572 & 0.116 \\
\hline Cage & 0.762101 & 0.807912 & 1984.811 & 0.943 & 0.346 \\
\hline Cage $^{2}$ & -0.015896 & 0.015617 & 1983.452 & -1.018 & 0.309 \\
\hline \multicolumn{6}{|l|}{ 70-74 years } \\
\hline Constant term & -0.151714 & 38.355675 & 930.311 & -0.004 & 0.997 \\
\hline$[$ Sex $=$ female $]$ & 0.132883 & 0.133375 & 1535.284 & 0.996 & 0.319 \\
\hline Calendar year & 0.004731 & 0.012500 & 1203.265 & 0.379 & 0.705 \\
\hline Cage & 0.066684 & 1.787135 & 737.826 & 0.037 & 0.970 \\
\hline Cage $^{2}$ & -0.002226 & 0.029030 & 738.639 & -0.077 & 0.939 \\
\hline \multicolumn{6}{|l|}{$75-79$ years } \\
\hline Constant term & -88.263384 & 87.934236 & 243.989 & -1.004 & 0.316 \\
\hline$[$ Sex $=$ female $]$ & -0.080334 & 0.240411 & 417.653 & -0.334 & 0.738 \\
\hline Calendar year & -0.000163 & 0.024665 & 404.215 & -0.007 & 0.995 \\
\hline Cage & 5.573218 & 4.115196 & 164.098 & 1.354 & 0.178 \\
\hline Cage $^{2}$ & -0.079425 & 0.057402 & 161.312 & -1.384 & 0.168 \\
\hline \multicolumn{6}{|l|}{ 80-94 years } \\
\hline Constant term & 269.841277 & 253.884634 & 29.281 & 1.063 & 0.297 \\
\hline$[$ Sex $=$ female $]$ & -0.116419 & 0.532094 & 69.045 & -0.219 & 0.827 \\
\hline Calendar year & -0.117305 & 0.057611 & 70.281 & -2.036 & 0.046 \\
\hline Cage & -1.608711 & 10.503836 & 28.890 & -0.153 & 0.879 \\
\hline Cage $^{2}$ & 0.024360 & 0.128926 & 29.518 & 0.189 & 0.851 \\
\hline \multicolumn{6}{|l|}{$85-89$ years } \\
\hline Constant term & 249.257018 & 1030.257290 & 15.000 & 0.242 & 0.812 \\
\hline$[$ Sex $=$ female $]$ & -1.029095 & 0.987104 & 15.000 & -1.043 & 0.314 \\
\hline Calendar year & 0.103347 & 0.108753 & 15.000 & 0.950 & 0.357 \\
\hline Cage & -18.560493 & 42.777174 & 15.000 & -0.434 & 0.671 \\
\hline Cage $^{2}$ & 0.192158 & 0.470681 & 15.000 & 0.408 & 0.689 \\
\hline
\end{tabular}

and male $\left(\mathrm{r}^{2}=0.06, \mathrm{p}=0.34\right)$ half-marathoners, age remained unchanged across years.

\section{Discussion}

This study intended to compare participation, performance and age of half-marathoners and marathoners competing in Switzerland between 1999 and 2014. The most important findings were: (1) more athletes competed in half-marathons than in marathons, (2) women were running faster than men in both half-marathons and marathons, (3) half-marathoners were running slower than marathoners, and (4) half-marathoners were younger than marathoners.

\section{Higher participation in half-marathons compared to marathons}

A first important finding was that 12.3 times more women and 7.5 times more men competed in half-marathon running than in marathon running, respectively; that was, an overall 8.7 half-marathon to marathon runner's ratio. This ratio was quite higher than the ratio of 3.71 which can be calculated from the data of the USA (www.runningusa.org/2015-national-runner-survey) for the year 2014 .

Thus, this ratio might vary from country to country and by gender, as a higher ratio was observed in women. For example, in the USA, the percentages of female and 


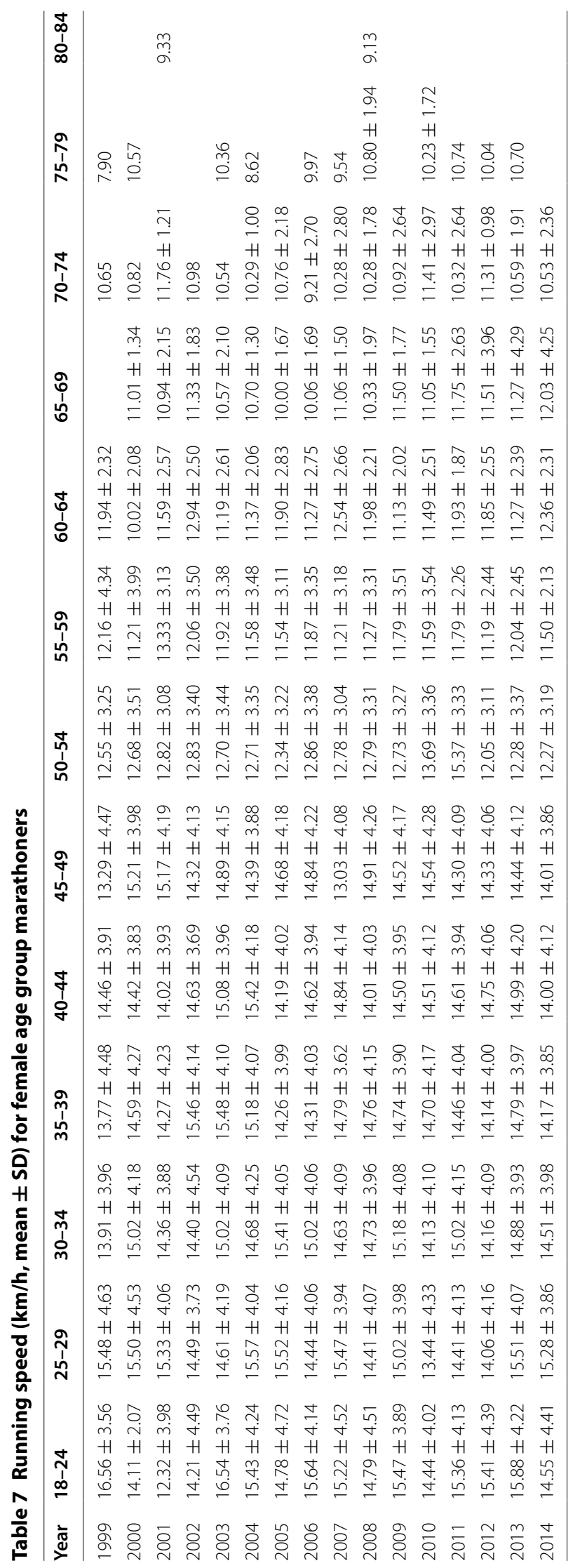




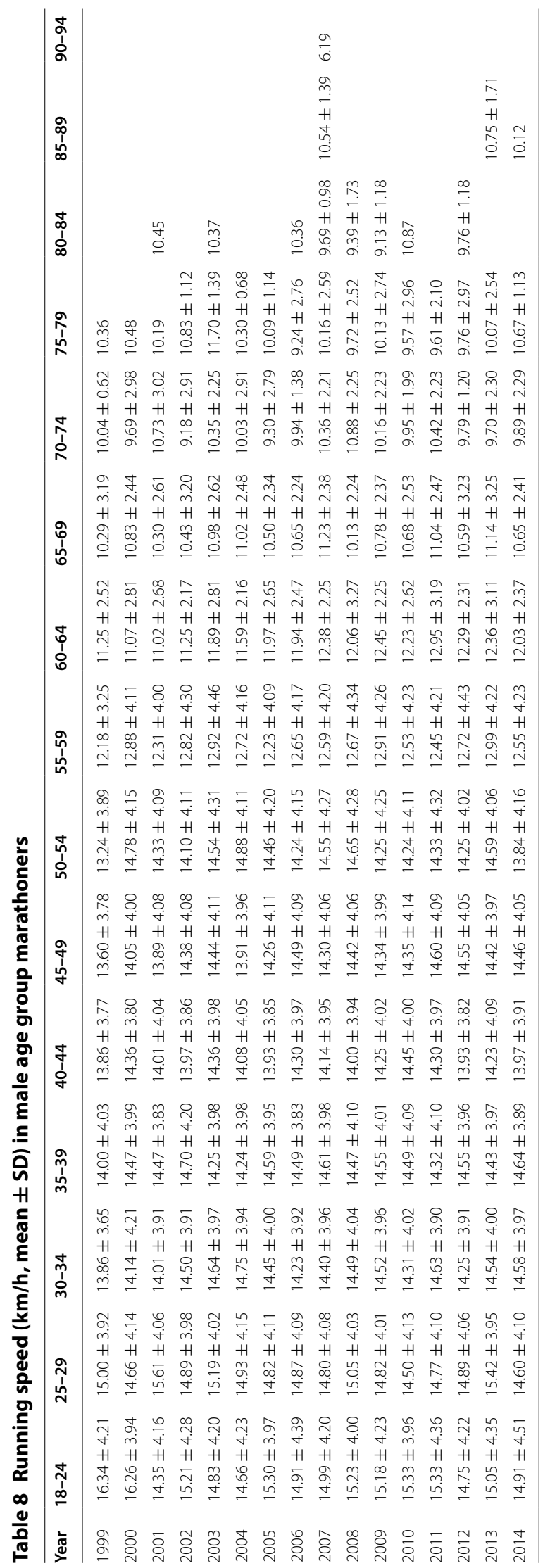


Table 9 Results of the mixed-effects regression analyses for running speed in marathon

\begin{tabular}{|c|c|c|c|c|c|}
\hline Parameter & Estimate & SE & df & $t$ & $p$ \\
\hline \multicolumn{6}{|l|}{$18-24$ years } \\
\hline Constant term & 54.963233 & 45.949509 & 1642.807 & 1.196 & 0.232 \\
\hline$[$ Sex $=$ female $]$ & -0.010120 & 0.247367 & 1808.569 & -0.041 & 0.967 \\
\hline Calendar year & -0.012919 & 0.022737 & 1694.390 & -0.568 & 0.570 \\
\hline Cage & 1.431063 & 0.966068 & 1517.761 & 1.481 & 0.139 \\
\hline Cage $^{2}$ & 0.036707 & 0.024531 & 1541.276 & 1.496 & 0.135 \\
\hline \multicolumn{6}{|l|}{$25-29$ years } \\
\hline Constant term & 35.772472 & 29.275418 & 3362.866 & 1.222 & 0.222 \\
\hline$[$ Sex $=$ female $]$ & 0.020400 & 0.169711 & 3644.906 & 0.120 & 0.904 \\
\hline Calendar year & -0.013980 & 0.014333 & 3395.444 & -0.975 & 0.329 \\
\hline Cage & -1.058839 & 0.842978 & 2700.446 & -1.256 & 0.209 \\
\hline Cage $^{2}$ & -0.037775 & 0.030204 & 2697.380 & -1.251 & 0.211 \\
\hline \multicolumn{6}{|l|}{ 30-34 years } \\
\hline Constant term & 18.697561 & 21.843554 & 4930.863 & 0.856 & 0.392 \\
\hline$[$ Sex $=$ female $]$ & 0.190907 & 0.136155 & 5546.306 & 1.402 & 0.161 \\
\hline Calendar year & -0.003024 & 0.010843 & 4923.040 & -0.279 & 0.780 \\
\hline Cage & -0.481635 & 0.395727 & 3466.504 & -1.217 & 0.224 \\
\hline Cage $^{2}$ & -0.027416 & 0.022019 & 3473.265 & -1.245 & 0.213 \\
\hline \multicolumn{6}{|l|}{ 35-39 years } \\
\hline Constant term & 24.985172 & 18.419663 & 5809.676 & 1.356 & 0.175 \\
\hline$[$ Sex $=$ female $]$ & 0.117198 & 0.120178 & 7035.058 & 0.975 & 0.329 \\
\hline Calendar year & -0.005163 & 0.009176 & 5811.848 & -0.563 & 0.574 \\
\hline Cage & -0.070901 & 0.146207 & 4084.836 & -0.485 & 0.628 \\
\hline Cage $^{2}$ & -0.010708 & 0.018256 & 4116.190 & -0.587 & 0.558 \\
\hline \multicolumn{6}{|l|}{ 40-44 years } \\
\hline Constant term & 24.560923 & 16.551235 & 6983.393 & 1.484 & 0.138 \\
\hline$[$ Sex $=$ female $]$ & 0.326742 & 0.110619 & 8241.252 & 2.954 & 0.003 \\
\hline Calendar year & -0.005067 & 0.008243 & 6983.203 & -0.615 & 0.539 \\
\hline Cage & 0.004824 & 0.037083 & 4870.786 & 0.130 & 0.896 \\
\hline Cage $^{2}$ & 0.008351 & 0.016000 & 4750.139 & 0.522 & 0.602 \\
\hline \multicolumn{6}{|l|}{ 45-49 years } \\
\hline Constant term & -18.776608 & 18.688413 & 6055.809 & -1.005 & 0.315 \\
\hline$[$ Sex $=$ female $]$ & 0.198713 & 0.121281 & 7183.064 & 1.638 & 0.101 \\
\hline Calendar year & 0.016356 & 0.009307 & 6056.803 & 1.757 & 0.079 \\
\hline Cage & 0.142027 & 0.219921 & 4077.826 & 0.646 & 0.518 \\
\hline Cage $^{2}$ & -0.008649 & 0.018296 & 4050.227 & -0.473 & 0.636 \\
\hline \multicolumn{6}{|l|}{ 50-54 years } \\
\hline Constant term & 35.122855 & 22.752641 & 3731.765 & 1.544 & 0.123 \\
\hline$[$ Sex $=$ female $]$ & 0.0376662 & 0.146151 & 5053.697 & 2.577 & 0.010 \\
\hline Calendar year & -0.013442 & 0.011249 & 3750.114 & -1.195 & 0.232 \\
\hline Cage & 1.138201 & 0.493626 & 2678.872 & 2.306 & 0.021 \\
\hline Cage $^{2}$ & -0.048973 & 0.022539 & 2671.667 & -2.173 & 0.030 \\
\hline \multicolumn{6}{|l|}{ 55-59 years } \\
\hline Constant term & 28.143217 & 31.538937 & 1687.963 & 0.892 & 0.372 \\
\hline$[$ Sex $=$ female $]$ & 0.426938 & 0.196830 & 2906.532 & 2.169 & 0.030 \\
\hline Calendar year & -0.007188 & 0.015146 & 1716.634 & -0.475 & 0.635 \\
\hline Cage & 0.096076 & 0.937454 & 1078.711 & 0.102 & 0.918 \\
\hline Cage $^{2}$ & -0.001525 & 0.029369 & 1070.383 & -0.052 & 0.959 \\
\hline
\end{tabular}

Table 9 continued

\begin{tabular}{|c|c|c|c|c|c|}
\hline Parameter & Estimate & SE & df & $\mathbf{t}$ & $p$ \\
\hline \multicolumn{6}{|l|}{ 60-64 years } \\
\hline Constant term & -42.833272 & 51.553601 & 1076.017 & -0.831 & 0.406 \\
\hline$[$ Sex $=$ female $]$ & 0.496350 & 0.272019 & 1574.678 & 1.825 & 0.068 \\
\hline Calendar year & 0.026103 & 0.023597 & 1184.052 & 1.106 & 0.269 \\
\hline Cage & 0.567461 & 1.978831 & 738.994 & 0.287 & 0.774 \\
\hline Cage $^{2}$ & -0.014553 & 0.047523 & 749.129 & -0.306 & 0.760 \\
\hline \multicolumn{6}{|l|}{$65-69$ years } \\
\hline Constant term & -59.473939 & 83.366094 & 418.223 & -0.713 & 0.476 \\
\hline$[$ Sex $=$ female $]$ & -0.231287 & 0.381408 & 737.633 & -0.606 & 0.544 \\
\hline Calendar year & 0.009479 & 0.034912 & 567.095 & 0.272 & 0.786 \\
\hline Cage & 4.275632 & 3.531786 & 262.482 & 1.211 & 0.227 \\
\hline Cage $^{2}$ & -0.081406 & 0.068094 & 260.904 & -1.195 & 0.233 \\
\hline \multicolumn{6}{|l|}{$70-74$ years } \\
\hline Constant term & -12.845200 & 143.465772 & 182.815 & -0.090 & 0.929 \\
\hline$[$ Sex $=$ female $]$ & -0.702660 & 0.576353 & 306.689 & -1.219 & 0.224 \\
\hline Calendar year & -0.065044 & 0.051496 & 257.801 & -1.263 & 0.208 \\
\hline Cage & 10.243172 & 6.298387 & 109.487 & 1.626 & 0.107 \\
\hline Cage $^{2}$ & -0.163818 & 0.102047 & 109.268 & -1.605 & 0.111 \\
\hline \multicolumn{6}{|l|}{$75-79$ years } \\
\hline Constant term & -60.658516 & 305.872037 & 10.992 & -0.198 & 0.846 \\
\hline$[$ Sex $=$ female $]$ & -0.568041 & 1.199866 & 84.162 & -0.473 & 0.637 \\
\hline Calendar year & -0.087646 & 0.099109 & 43.052 & -0.884 & 0.381 \\
\hline Cage & 13.998502 & 14.954195 & 14.595 & 0.936 & 0.364 \\
\hline Cage $^{2}$ & -0.193455 & 0.209581 & 14.944 & -0.923 & 0.371 \\
\hline \multicolumn{6}{|l|}{$80-84$ years } \\
\hline Constant term & -1994.108804 & 706.076437 & 15.000 & -2.824 & 0.013 \\
\hline$[$ Sex $=$ female $]$ & -1.193533 & 1.235529 & 15.000 & -0.966 & 0.349 \\
\hline Calendar year & -0.064119 & 0.145369 & 15.000 & -0.441 & 0.665 \\
\hline Cage & 104.090501 & 24.280352 & 15.000 & 4.287 & 0.001 \\
\hline Cage $^{2}$ & -1.263960 & 0.295597 & 15.000 & -4.276 & 0.001 \\
\hline \multicolumn{6}{|l|}{$85-89$ years } \\
\hline Constant term & -275.995974 & 516.465002 & 6.000 & -0.534 & 0.612 \\
\hline$[$ Sex $=$ female $]$ & 0 & 0 & & & \\
\hline Calendar year & 0.043759 & 0.245249 & 6.000 & 0.178 & 0.864 \\
\hline Cage & 4.599196 & 1.543800 & 6.000 & 2.979 & 0.025 \\
\hline Cage $^{2}$ & 0 & 0 & & & \\
\hline
\end{tabular}

male half-marathoners in 2014 were 61 and $39 \%$, respectively (www.runningusa.org/half-marathon-report-2015). For marathoners in the same year and the same country, the percentages were, however, 43 and $57 \%$, respectively (www.runningusa.org/marathon-report-2015). In other terms, 1.56 times more women competed in half-marathon running, but 1.32 times more men in marathon running in 2014 in the USA. When we compare the 2014 data of the USA to the data from 1999-2014 in Switzerland, 10.2 times more marathoners competed in the USA $(550,637)$ compared to Switzerland $(53,694)$. Considering the sexes, there were 23.2 times more women and 7.2 
Table 10 Sex difference (\%) in running speed in age group half-marathoners

\begin{tabular}{|c|c|c|c|c|c|c|c|c|c|c|c|c|c|c|}
\hline Year & $18-24$ & $25-29$ & $30-34$ & $35-39$ & $40-44$ & $45-49$ & $50-54$ & $55-59$ & $60-64$ & $65-69$ & $70-74$ & $75-79$ & $80-84$ & $85-89$ \\
\hline 1999 & 1.4 & -0.8 & 1.7 & 3.0 & 2.8 & 2.2 & 6.3 & 3.0 & -1.3 & -3.9 & -11.1 & -2.3 & & -49.0 \\
\hline 2000 & 3.5 & 0.7 & 1.1 & 3.7 & 3.3 & 0.3 & 1.6 & 2.9 & 9.4 & 4.4 & 10.4 & -7.2 & & \\
\hline 2001 & -1.1 & 3.0 & 0.6 & 2.4 & 2.8 & -0.7 & 0.4 & 0.8 & 2.0 & -0.8 & -11.1 & 12.8 & & \\
\hline 2002 & -0.2 & 2.9 & 4.0 & 3.9 & 1.3 & 0.5 & 3.1 & 0.7 & 0.4 & 3.3 & 6.3 & 1.5 & 2.2 & \\
\hline 2003 & 1.2 & 1.5 & 2.3 & 1.7 & -1.6 & 3.5 & 2.5 & 1.5 & 5.3 & 6.7 & 7.4 & 13.7 & & \\
\hline 2004 & 1.7 & 3.4 & 1.3 & 2.6 & 0.8 & 0.3 & 2.7 & 1.3 & -1.3 & -2.8 & 15.0 & -2.4 & 5.0 & 13.5 \\
\hline 2005 & -3.7 & 0.5 & 1.4 & 1.6 & 1.1 & 1.1 & -0.6 & -1.8 & 0.1 & 1.2 & -2.3 & 21.8 & 1.5 & \\
\hline 2006 & -0.9 & -2.2 & 0.1 & 2.5 & -0.06 & 0.9 & 0.7 & 0.09 & -1.8 & -3.1 & 11.7 & -25.2 & & \\
\hline 2007 & -0.8 & -0.2 & -0.6 & 1.1 & -3.2 & -0.8 & -0.6 & 0.2 & -1.4 & -1.7 & -11.8 & -0.6 & -7.9 & -22.7 \\
\hline 2008 & -0.2 & -0.8 & 1.6 & 1.6 & -0.6 & 1.4 & -0.7 & -0.7 & -1.0 & 2.6 & 3.1 & -0.2 & -34.2 & \\
\hline 2009 & -0.4 & -0.1 & 1.9 & 0.03 & 0.6 & 1.5 & 1.1 & 2.1 & 0.9 & 3.3 & 3.8 & 28.9 & -22.4 & \\
\hline 2010 & 0.5 & 0.7 & 2.4 & 0.03 & 1.2 & -0.5 & 1.7 & 0.7 & 1.2 & 4.2 & 3.4 & -10.4 & -9.3 & \\
\hline 2011 & -0.8 & 0.9 & 1.5 & 1.6 & 1.5 & 1.3 & -1.4 & 1.2 & 0.1 & 5.8 & -6.2 & -7.3 & 9.2 & \\
\hline 2012 & 2.4 & -1.1 & 2.3 & 0.4 & 0.8 & 0.5 & 0.5 & 2.8 & 0.4 & 3.6 & 0.7 & 2.1 & -11.6 & 7.9 \\
\hline 2013 & 1.5 & 1.5 & -0.3 & -0.4 & 0.4 & -0.1 & 0.4 & 0.4 & 1.7 & -0.6 & 4.1 & -3.1 & -3.4 & \\
\hline 2014 & 0.5 & -0.0 & 0.1 & 0.1 & -0.02 & -0.1 & -0.5 & -0.2 & 0.01 & 1.1 & 0.07 & -9.0 & & \\
\hline$r^{2}$ & 0.001 & 0.08 & 0.06 & 0.73 & 0.145 & 0.06 & 0.39 & 0.07 & 0.08 & 0.04 & 0.0001 & 0.02 & 0.05 & 0.44 \\
\hline$p$ & 0.89 & 0.26 & 0.34 & $<0.0001$ & 0.14 & 0.33 & 0.0092 & 0.31 & 0.27 & 0.42 & 0.97 & 0.55 & 0.51 & 0.33 \\
\hline
\end{tabular}

Table 11 Sex difference (\%) in running speed in age group marathoners

\begin{tabular}{|c|c|c|c|c|c|c|c|c|c|c|c|c|c|}
\hline Year & $18-24$ & $25-29$ & $30-34$ & $35-39$ & $40-44$ & $45-49$ & 50-54 & $55-59$ & $60-64$ & $65-69$ & $70-74$ & $75-79$ & $80-84$ \\
\hline 1999 & 1.3 & 3.1 & 0.4 & -1.6 & 4.3 & -2.2 & 9.9 & -13.3 & 10.4 & & -7.7 & -59.1 & \\
\hline 2000 & -37.8 & 5.7 & 6.2 & 0.8 & 0.4 & 8.2 & -0.6 & 2.2 & -14.5 & -40.4 & 28.0 & 19.9 & \\
\hline 2001 & -14.1 & -1.8 & 2.5 & -1.3 & 0.1 & 9.1 & 3.4 & 14.0 & -9.5 & -14.4 & -16.7 & & -0.6 \\
\hline 2002 & -6.5 & -2.6 & -0.6 & 5.1 & 4.7 & -0.3 & 5.1 & -5.1 & -17.4 & -0.6 & -13.6 & & \\
\hline 2003 & 11.4 & -3.8 & 2.5 & 8.6 & 5.0 & 3.1 & 1.1 & -6.6 & 8.7 & 10.5 & -17.1 & 56.9 & \\
\hline 2004 & 5.2 & 4.3 & -0.4 & 6.6 & 9.4 & 3.4 & -1.1 & 5.8 & 12.1 & -2.1 & 30.3 & -55.3 & \\
\hline 2005 & -3.4 & 4.6 & 6.6 & -2.2 & 1.9 & 3.0 & -0.8 & 9.2 & 6.1 & 10.3 & -27.8 & & \\
\hline 2006 & 4.9 & -2.9 & 5.5 & -1.2 & 2.2 & 2.4 & 4.3 & 8.2 & 2.2 & 2.6 & -24.9 & 15.8 & \\
\hline 2007 & 1.5 & 4.5 & 1.6 & 1.2 & 4.9 & 5.1 & 1.6 & 4.2 & -5.8 & -1.1 & 5.6 & -44.4 & \\
\hline 2008 & -2.9 & -4.2 & 1.6 & 1.9 & 0.1 & 3.3 & 0.9 & 4.0 & -0.5 & 1.2 & 15.1 & -16.4 & -18.7 \\
\hline 2009 & 1.8 & 1.3 & 4.5 & 1.3 & 1.8 & 1.2 & 3.3 & 5.9 & 4.4 & -1.9 & -1.5 & & \\
\hline 2010 & -5.8 & -7.3 & -1.2 & 1.4 & 0.4 & 1.3 & 10.1 & 7.2 & 8.3 & 2.3 & -32.6 & 45.1 & \\
\hline 2011 & 0.2 & -2.4 & 2.6 & 0.9 & 2.1 & -2.0 & 7.2 & 2.3 & -0.1 & -1.8 & 13.1 & 21.4 & \\
\hline 2012 & 4.4 & -5.6 & -0.6 & -2.7 & 5.9 & -1.4 & 5.6 & -3.6 & 10.1 & -13.3 & -7.8 & 13.6 & \\
\hline 2013 & 5.5 & 0.5 & 2.3 & 2.4 & 5.3 & 0.1 & 4.7 & 0.3 & -0.6 & -11.5 & 18.4 & 43.0 & \\
\hline 2014 & -2.4 & 4.6 & -0.5 & -3.2 & 0.1 & -3.1 & 3.0 & 6.5 & 8.8 & 2.6 & -9.1 & & \\
\hline$r^{2}$ & 0.15 & 0.05 & 0.05 & 0.05 & 0.006 & 0.28 & 0.04 & 0.05 & 0.12 & 0.06 & 0.0009 & 0.16 & 1 \\
\hline$p$ & 0.13 & 0.37 & 0.36 & 0.36 & 0.76 & 0.033 & 0.45 & 0.39 & 0.18 & 0.37 & 0.91 & 0.21 & \\
\hline
\end{tabular}

times more men in the USA than in Switzerland. For halfmarathon running, there were 4.5 times more athletes in the USA $(2046,600)$ compared to Switzerland $(454,324)$. There were 9.9 times more women and 2.4 times more men in the USA compared to Switzerland. Considering the data of Leyk et al. (2007) investigating 65 half-marathons and 69 marathons held in Germany between 2003 and 2005 , a total of 156,717 men and 144,640 women were considered with a ratio of 1.08 . There were 4.85 and 2.6 times more men in marathons and half-marathons, respectively (Leyk et al. 2007). When we compare their data to the data from Switzerland, we had 2.9 times fewer marathoners, but 3.1 times more half-marathoners. In details, we considered 2.6 times fewer male $(43,489$ 


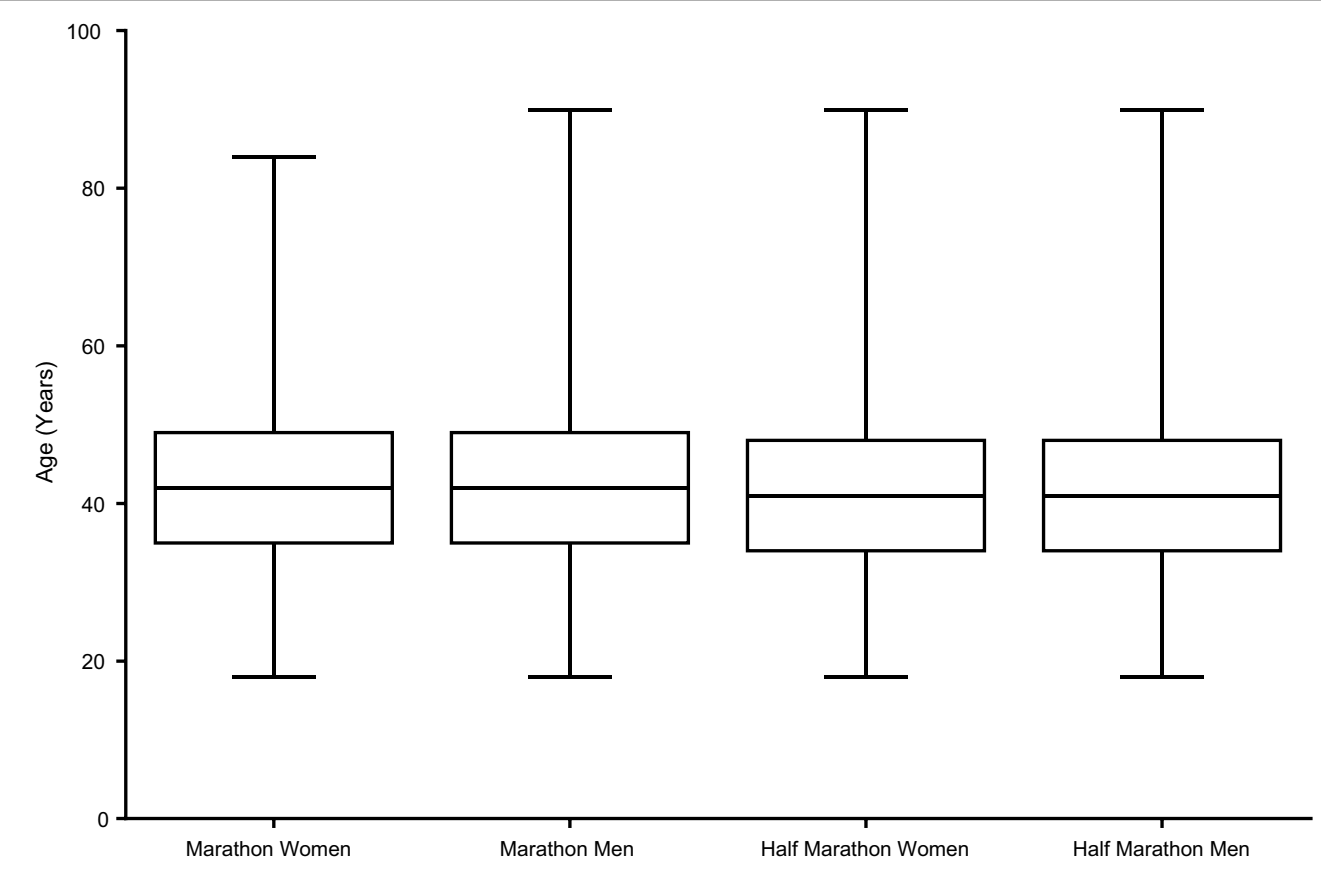

Fig. 3 Box-whisker-plot for age in female and male half-marathoners and marathoners. Data are presented as mean $\pm 95 \%$ confidence interval
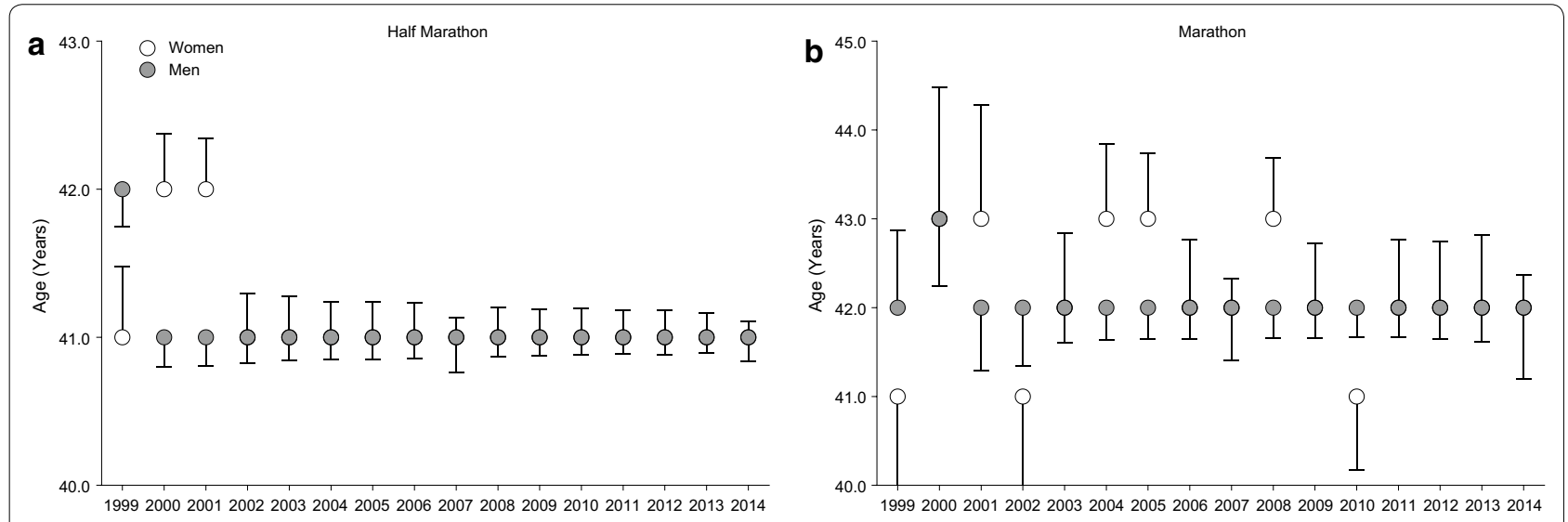

Fig. 4 Age across years in half-marathoners and marathoners. Data are presented as mean \pm SD. From 2002 to 2014, data for women are hidden behind data for men

vs. 129,929$)$ and 2.98 fewer female $(10,205$ vs. 26,788$)$ marathoners. For half-marathoners, we investigated 3.1 times more female half-marathoners $(125,894$ vs. 39,998$)$ and 3.1 times more male half-marathoners (328,430 vs. 104,042). Obviously, the data set and the country seems to have an influence on the participation trends in halfmarathon and marathon running.

An interesting observation was that participation increased across years in both half-marathon and marathon running. When the Swiss data were investigated from 2000 to 2010, the number of half-marathoners increased significantly for both men and women. In contrast, the number of male and female full marathoners increased until 2005 only and decreased thereafter (Anthony et al. 2014). Most probably, after 2010, a new increase (hype) in marathon running occurred in Switzerland, which might also explain the better performance in marathon running compared to half-marathon running.

\section{Women were faster than men}

A second important and unexpected finding was that women were significantly faster than men in both 
half-marathon and marathon running. However, the differences were very small, but still significant. This finding was not in agreement with a previous study, in which female long-distance runners were slower by 22.5 and $20 \%$ in half-marathon and marathon running, respectively, than men (Leyk et al. 2007). A potential explanation could the sample size. While Leyk et al. (2007) considered 405,515 race times, we analyzed 508,108 race times (i.e. $25.3 \%$ more athletes).

An explanation of a superior performance in men might be their training characteristics, as a research on the these characteristics of the 2004 USA Olympic marathon trials qualifiers showed that men and women ran 75 and $68 \%$ of their weekly training distance, respectively, below marathon race pace, and men had more years of sports experience, ran more often and ran farther (Karp 2007). It has been shown that in men, the mean weekly running distance, the minimum distance run per week, the maximum distance run per week, the mean weekly hours of running, the number of running training sessions per week, and the mean speed of the training sessions were significantly and negatively related to total race time, but not in women (Knechtle et al. 2010).

A potential explanation for the disparate findings in Leyk et al. (2007) and our findings for female and male running performance could be the kind of analysis. While Leyk et al. (2007) compared in their study the top ten half-marathon and marathon race times in women and men, women were on average $20 \%$ slower in marathon and $22.5 \%$ slower in half-marathon. In the present study, however, women were faster than men when all recorded women and men were considered for data analysis. While Leyk et al. (2007) investigated 104,042 male and 39,998 female half-marathoners and 129,929 male and 26,788 female marathoners, our numbers were 328,430 male and 125,894 female half-marathoners and 43,489 male and 10,205 female marathoners. In fact, we considered 3.15 times more half-marathoners (i.e. 3.15 times more men and 3.14 times more women) but 2.9 times fewer marathoners (i.e. 2.98 fewer men and 2.62 times fewer women).

The considerably higher number of half-marathoners might explain why they were significantly slower than marathoners. In a large number of athletes, also slow to very slow runners are included. Similarly, the lower number of marathoners might be a selection of faster runners. Most probably, more recreational runners compete in Switzerland in half-marathons and more elite runners in marathons. This assumption might be supported by the data from 2014 in the USA where 3.71 more female and male runners competed in half-marathons (www.runningusa.org/half-marathon-report-2015) compared to marathons (www.runningusa.org/marathon-report-2015).
There were more women $(61 \%, 1,248,426)$ than men $(39 \%, 798,174)$ competing in half-marathons but more men $(57 \%, 313,863)$ than women $(43 \%, 236,774)$ in marathons. In half-marathon races, women (2:21 h:min) were running 0:19 h:min (7.42 \%) slower than men (2:02). In marathon races, women ( $4: 19 \mathrm{~h}: \mathrm{min})$ were running 0:25 h:min (11.36 \%) slower than men (4:19 h:min). A further explanation for the different findings between Leyk et al. (2007) and our findings could be the period of time. While Leyk et al. (2007) considered marathons in Germany held from 2003 to 2005 (i.e. 3 years) we included marathons in Switzerland held from 1999 to 2014 (i.e. 15 years). Across years, women were able to improve their running performance.

Nevertheless, the present study was not the first one to observe a superior performance in women during an endurance event. Recently, a superior performance of women was noticed in ultra-distance swimming (Knechtle et al. 2014, 2015a), which might be attributed to anthropometric characteristics such as body fat.

\section{Half-marathoners are running slower than marathoners}

A third important finding was that female and male halfmarathoners were running slower than female and male marathoners. This might be explained by their pre-race preparation, their sport experience and their competitive level. In a field study comparing 147 recreational male half-marathoners and 126 recreational male marathoners, the half-marathoners were running for fewer years, completed less weekly running kilometers, they were running fewer hours per week, completed fewer training sessions, achieved fewer kilometers per training session, and invested fewer minutes per training session compared to the marathoners (Zillmann et al. 2013). However, in that study, the half-marathoners $(12.2 \pm 1.9 \mathrm{~km} / \mathrm{h})$ were running significantly faster than the marathoners $(11.1 \pm 1.4 \mathrm{~km} / \mathrm{h})$. This might be explained by the fact that the subjects could participate in that study voluntarily and the interests to take part in such an investigation might be different for half-marathoners and marathoners.

Another potential bias could be the race fee and/or average yearly income in USA, Switzerland and Germany. The race fee in a half-marathon is lower than in a full marathon. For example, the entry fee for running the half-marathon in 'Lausanne Marathon' is 52 Swiss Francs, but 80 Swiss Francs for running the full marathon (http:// de.lausanne-marathon.com/inscription/inscriptions/ prix-categories/). While the annual income is higher in the USA compared to Germany, the income in Switzerland is higher compared to Germany. In Switzerland, the average household net-adjusted disposable income per capita is USD 33, 491 a year (www.oecdbetterlifeindex. 
org/countries/switzerland). In comparison, the average household net-adjusted disposable income per capita is USD 31,252 in Germany (www.oecdbetterlifeindex.org/ countries/germany). In the USA, however, the income is higher with USD 41,355 (www.oecdbetterlifeindex.org/ countries/united-states).

Differences in performance between half-marathoners and marathoners might be due to differences in anthropometric characteristics. For instance, with regards to anthropometric characteristics, male half-marathoners were heavier with longer legs, thicker upper arms and thigh, and higher skinfold thicknesses, body fat percentage and skeletal muscle mass compared to male marathoners (Zillmann et al. 2013). Compared to ultramarathoners, female half-marathoners were younger, heavier, reported a lower training volume and had a lower incidence of bone stress injury (Micklesfield et al. 2007). Race time in half-marathon running might be predicted by body mass index, resting heart rate, training volume and sport experience (Campbell, 1985). In addition to anthropometric and training characteristics, the performance in half-marathon running has been shown to be influenced by certain physiological parameters. In a small group of female and male half-marathoners, their race speed corresponded to $\sim 79 \%$ of $\mathrm{VO}_{2}$ max and their race time correlated to $\mathrm{VO}_{2} \max$ and running speed at blood lactate concentration $4 \mathrm{mmol} \cdot \mathrm{l}^{-1}$ (Williams and Nute, 1983). In a comparison of female middle- and long-distance runners, the race time in half-marathon running correlated to body mass, but not to $\mathrm{VO}_{2} \max$, anaerobic threshold or running economy (Nurmekivi et al. 1998). Performance in marathon running has been shown to be limited by the rate of aerobic metabolism of a limited amount of carbohydrate energy and the velocity that can be maintained without developing hyperthermia (Coyle, 2007). Serum leptin, which decreases in the blood when the energy balance is negative, lowered after an ultra-marathon race, but not after a half-marathon (Zaccaria et al. 2002). In a genetic study of ACE I/D polymorphism, no association between half-marathoners and the ACE genotype was found, whereas an increase of the I/I genotype incidence in the successful marathoners was observed (Hruskovicová et al. 2006).

\section{Half-marathoners were younger than marathoners}

A fourth important finding was that female and male half-marathoners were younger than female and male marathoners. Within a race distance, no differences were found between the sexes. These findings were different to the study subjects in the field study of Zillmann et al. (2013). There, the age of male half-marathoners was $40.2 \pm 10.1$ and $42.8 \pm 10.8$ years for male marathoners.
However, the difference of $\sim 2.6$ years was not statistically significant.

Similarly, in US-American half-marathoners (www. runningusa.org/half-marathon-report-2015) and marathoners (www.runningusa.org/marathon-report-2015), the ages were different. In marathoners competing in the USA in 2014, women ( 40.0 years) were on average $\sim 4.0$ years older than men ( $\sim 36.0$ years). In half-marathoners, women ( $\sim 36.0$ years) were $\sim 3.1$ years younger than men ( $\sim 39.1$ years). Therefore, female half-marathoners were $\sim 4.0$ years younger than female marathoners, and male half-marathoners were $\sim 3.1$ years older than male marathoners. The differences might be explained that in the study of Zillmann et al. (2013) only athletes competing in one marathon in Switzerland were examined and in the statistic report in the USA, the median age of all successful finishers was provided (www.runningusa.org/ statistics).

A further observation was that women were at about the same age than men in marathon ( $\sim 42$ years $)$ and half-marathon ( $\sim 41$ years) running. However, women in marathon running were significantly older than women in half-marathon running and men in marathon running were significantly older than women in half-marathon running. Generally, elite marathoners are considerably younger than the athletes in the present sample. In the study of Hunter et al. (2011) investigating the first five placed women and men competing in marathons of the 'World Marathon Majors Series' in Berlin, Boston, Chicago, London, New York City, the International Athletic Association Federation (IAAF) World Championships, and the Olympic Games, women (29.8 \pm 4.2 years) were older than men ( $28.9 \pm 3.8$ years), but for only two (i.e. Chicago and London) of the seven marathons with no sex difference in age for the marathons held in Berlin, Boston, New York City, and at the IAAF World Championships and the Olympic Games.

The age of the best marathon performance is, however, higher in recreational runners. In a study investigating male amateur runners competing in the Stockholm Marathon between 1979 and 2014, marathon race performance of the average runner improved up to age of $34.3 \pm 2.6$ years. After that age, the marathon race performance started to decline (Lehto 2015). The differences between the age might be explained in that Hunter et al. (2011) investigated elite marathoners competing at world class level while we considered all successful finishers in half-marathon and marathon running.

\section{Limitations}

A limitation of the present study was the lack of information with regards to the competitive level of runners, i.e. 
whether they were elite or recreational athletes. The possibility that women were more competitive than men and overall marathoners more competitive than overall halfmarathoners cannot be excluded and might account for the better performance in women and in marathoners, respectively. On the other hand, data on more than half a million runners were examined in this study which was one of the largest samples of half-marathoners and marathoners ever studied.

\section{Conclusions}

In summary, for runners competing between 1999 and 2014 in Swiss half-marathons and marathons, (1) more athletes competed in half-marathons than in marathons, (2) women were running faster than men, (3) half-marathoners were running slower than marathoners, and (4) half-marathoners were younger than marathoners.

\section{Authors' contributions}

BK and $M Z$ collected all data, BK and PN drafted the manuscript, CR and PN performed the statistical analyses, CR and TR participated in the design and coordination and helped drafting the manuscript. All authors read and approved the final manuscript.

\section{Author details \\ ${ }^{1}$ Gesundheitszentrum St. Gallen, St. Gallen, Switzerland. ${ }^{2}$ Institute of Primary Care, University of Zurich, Zurich, Switzerland. ${ }^{3}$ Department of Physical and Cultural Education, Hellenic Army Academy, Athens, Greece.}

\section{Competing interests}

The authors declare that they have no competing interests.

Received: 23 December 2015 Accepted: 12 January 2016

Published online: 26 January 2016

\section{References}

Ahmadyar B, Rüst CA, Rosemann T, Knechtle B (2015) Participation and performance trends in elderly marathoners in four of the world's largest marathons during 2004-2011. Springerplus 4:465

Anthony D, Rüst CA, Cribari M, Rosemann T, Lepers R, Knechtle B (2014) Differences in participation and performance trends in age group half and full marathoners. Chin J Physiol 57:209-219

Campbell MJ (1985) Predicting running speed from a simple questionnaire. $\mathrm{Br}$ J Sports Med 19:142-144

Coyle EF (2007) Physiological regulation of marathon performance. Sports Med 37:306-311

De Gonzalo-Calvo D, Dávalos A, Montero A, García-González Á, Tyshkovska I, González-Medina A, Soares SM, Martínez-Camblor P, Casas-Agustench P, Rabadán M, Díaz-Martínez ÁE, Úbeda N, Iglesias-Gutiérrez E (2015) Circulating inflammatory miRNA signature in response to different doses of aerobic exercise. J Appl Physiol (1985) 119:124-134

Hart L (2013) Marathon-related cardiac arrest. Clin J Sport Med 23:409-410

Hruskovicová H, Dzurenková D, Selingerová M, Bohus B, Timkanicová B, Kovács $L$ (2006) The angiotensin converting enzyme I/D polymorphism in long distance runners. J Sports Med Phys Fitness 46:509-513
Hunter SK, Stevens AA, Magennis K, Skelton KW, Fauth M (2011) Is there a sex difference in the age of elite marathon runners? Med Sci Sports Exerc 43:656-664

Jassal DS, Moffat D, Krahn J, Ahmadie R, Fang T, Eschun G, Sharma S (2009) Cardiac injury markers in non-elite marathon runners. Int J Sports Med 30:75-79

Jokl P, Sethi PM, Cooper AJ (2004) Master's performance in the New York City Marathon 1983-1999. Br J Sports Med 38:408-412

Karp JR (2007) Training characteristics of qualifiers for the U.S. Olympic Marathon Trials. Int J Sports Physiol Perform 2:72-92

Kim JH, Malhotra R, Chiampas G, d'Hemecourt P, Troyanos C, Cianca J, Smith RN, Wang TJ, Roberts WO, Thompson PD, Baggish AL, Race Associated Cardiac Arrest Event Registry (RACER) Study Group (2012) Cardiac arrest during long-distance running races. N Engl J Med 366:130-140

Knechtle B, Knechtle P, Rosemann T, Senn O (2010) Sex differences in association of race performance, skin-fold thicknesses, and training variables for recreational half-marathon runners. Percept Mot Skills 111:653-668

Knechtle B, Rosemann T, Lepers R, Rüst CA (2014) Women outperform men in ultradistance swimming: the Manhattan Island Marathon Swim from 1983 to 2013. Int J Sports Physiol Perform 9:913-924

Knechtle B, Rosemann T, Rüst CA (2015a) Women cross the 'Catalina Channel' faster than men. Springerplus 4:332

Knechtle B, Rosemann T, Zingg MA, Rüst CA (2015b) Increase in participation but decrease in performance in age group mountain marathoners in the 'Jungfrau Marathon': a Swiss phenomenon? Springerplus 4:523

Lehto N (2015) Effects of age on marathon finishing time among male amateur runners in Stockholm Marathon 1979-2014. J Sport Health Sci. doi:10.1016/j.jshs.2015.01.008

Lepers R, Cattagni T (2012) Do older athletes reach limits in their performance during marathon running? Age (Dordr) 34:773-781

Leyk D, Erley O, Ridder D, Leurs M, Rüther T, Wunderlich M, Sievert A, Baum K, Essfeld D (2007) Age-related changes in marathon and half-marathon performances. Int J Sports Med 28:513-517

Micklesfield LK, Hugo J, Johnson C, Noakes TD, Lambert EV (2007) Factors associated with menstrual dysfunction and self-reported bone stress injuries in female runners in the ultra-and half-marathons of the two oceans. Br J Sports Med 41:679-683

Nurmekivi A, Lemberg H, Maaroos J, Lusti J, Jürimäe T (1998) Running performance and aerobic working capacity in female runners. Med Sport 51:221-225

Reihmane D, Jurka A, Tretjakovs P, Dela F (2013) Increase in IL-6, TNF-a, and MMP-9, but not sICAM-1 concentrations depends on exercise duration. Eur J Appl Physiol 113:851-858

Williams C, Nute ML (1983) Some physiological demands of a half-marathon race on recreational runners. Br J Sports Med 17:152-161

Zaccaria M, Ermolao A, Roi GS, Englaro P, Tegon G, Varnier M (2002) Leptin reduction after endurance races differing in duration and energy expenditure. Eur J Appl Physiol 87:108-111

Zillmann T, Knechtle B, Rüst CA, Knechtle P, Rosemann T, Lepers R (2013) Comparison of training and anthropometric characteristics between recreational male half-marathoners and marathoners. Chin J Physiol 56:138-146

\section{Submit your manuscript to a SpringerOpen ${ }^{\circ}$ journal and benefit from:}

- Convenient online submission

- Rigorous peer review

- Immediate publication on acceptance

- Open access: articles freely available online

- High visibility within the field

- Retaining the copyright to your article

Submit your next manuscript at $>$ springeropen.com 